\title{
1 Karrikin-sensing protein KAI2 is a new player in regulating root growth patterns
}

2

3

4 Stéphanie M. Swarbreck ${ }^{1 *}$, Yannick Guerringue ${ }^{1,2}$, Elsa Matthus ${ }^{1}$, Fiona J. C. Jamieson ${ }^{1,3}$ and 5 Julia M. Davies ${ }^{1}$

6

$7{ }^{1}$ Department of Plant Sciences, University of Cambridge, Cambridge, CB2 3EA, United 8 Kingdom

$9 \quad 2$ ENS de Lyon - Site Monod, Lyon, 69007, France

$10{ }^{3}$ Department of Plant Sciences, University of Oxford, South Parks Road, Oxford, OX1 3RB,

11 United Kingdom

$12{ }^{*}$ Corresponding author, ss2062@cam.ac.uk, 01223-748-980

13

14 Total word count: 6,192

15 Word count Introduction: 708

16 Word count Materials and Methods: 1,419

17 Word count Results: 2,557

18 Word count Discussion: 1,236

19 Total number of figures: 8

20 Total number of supplementary figures: 6 


\section{Summary}

Roots form highly complex systems varying in growth direction and branching pattern to forage for nutrients efficiently. Here mutations in the KAI2 (KARRIKIN INSENSITIVE) $\alpha / \beta$ fold hydrolase and the MAX2 (MORE AXILLARY GROWTH 2) F-box leucine-rich protein, which together perceive karrikins (smoke-derived butenolides), caused alteration in root growth direction (root skewing and waving) of Arabidopsis thaliana. This exaggerated root skewing was independent of endogenous strigolactone perception by the D14 $\alpha / \beta$-fold hydrolase and MAX2. Thus KAI2/MAX2's regulation of root growth may be through perception of endogenous KAI2-ligands, which have yet to be identified. Degradation targets of the KAI2/MAX2 complex, SMAX1 (SUPPRESSOR OF MAX2-1) and SMXL6,7,8 (SUPPRESSOR OF MAX2-1-LIKE) are also involved in the regulation of root skewing. Genetic data reveal a new potential target for degradation, as mutation in the SKS3 (SKU5 similar) but not the SKU5/SKS17 root plasma membrane glycoprotein suppresses the exaggerated root skewing induced by the lack of MAX2. In Arabidopsis thaliana therefore, the KAI2 karrikin-sensing protein acts to limit root skewing, and we propose a mechanism involving root radial expansion as the mutant's gravitropic and mechano-sensing responses remained largely unaffected.

\section{Introduction}

Roots grow in complex patterns that are highly relevant to their adaptation to different soil conditions and yet very difficult to investigate in this complex medium. Arabidopsis thaliana roots grown vertically on solid medium produce specific surface-dependent growth patterns described as skewing (deviation from vertical) and waving (Roy \& Bassham, 2014). Established differences amongst Arabidopsis ecotypes suggest that these patterns may reflect an adaptive response relevant to natural soil conditions (Vaughn \& Masson, 2011; Schultz et al., 2017).

While root skewing has been widely observed and reported, it is not fully understood and no model akin to that available for the root gravitropic response has been proposed (Darwin \& Darwin, 1880; Oliva \& Dunand, 2007; Roy \& Bassham, 2014). The characterization of Arabidopsis mutants has been critical in identifying genetic components that can govern root skewing and waving (Okada \& Shimura, 1990; Wang et al., 2011; Shih et al., 2014), which represent the integrated response to gravity, light and contact with the solid medium as the root 
56

57

tip grows on the surface of the agar (Thompson \& Holbrook, 2004). A skewing phenotype in mutants impaired in mechano-sensing such as feronia (Shih et al., 2014) or cml24 (Wang et al., 2011) supports a link between root skewing and thigmomorphogenesis (morphological change in response to mechanical stimulation from surface contact).

The role of plant hormones in root skewing and waving is poorly understood but auxins (Okada \& Shimura, 1990), ethylene (Buer et al., 2000; 2003), cytokinins (Kushwah et al., 2011) and brassinosteroids (Lanza et al., 2012) are implicated. Little is known of the role of a recently characterized set of phytohormones, strigolactones (SL, Roy \& Bassham, 2014) and related smoked-derived butenolides, karrikins (KAR, Flematti et al., 2015) or the as yet unidentified endogenous ligands of the KAI2 (KARRIKIN INSENSITIVE) karrikins receptor (KAI2 ligand, KL, Sun et al., 2016).

Many elements of the KAR/KL perception pathway have been elucidated and are either shared or related to components of the SL perception pathway. The current model suggests that KARs and KLs are perceived by binding the $\alpha / \beta$-fold hydrolase KAI2/D14-like protein (Waters et al., 2012; Bythell-Douglas et al., 2013), while SLs bind a related $\alpha / \beta$-fold hydrolase called D14 (Hamiaux et al., 2012; Chevalier et al., 2014; Yao et al., 2016). D14 can form a complex with MAX2 (MORE AXILLARY GROWTH2), a leucine-rich repeat F-box protein (Zhao et al., 2015; Yao et al., 2016). It has been hypothesised that KAI2 can also form such a complex with MAX2 though no physical interaction has been confirmed yet (Conn \& Nelson, 2016). In addition, the KAR-dependent degradation of KAI2 can occur independently from MAX2, independently of ubiquitination or the activity of the $26 \mathrm{~S}$ proteasome (Waters et al., 2015a). More recently heat-shock related proteins have been identified as degradation targets of MAX2 in rice (D53, Jiang et al., 2013; Zhou et al., 2013) and Arabidopsis (SMXL, SUPPRESSOR OF MAX2-1-LIKE, (Stanga et al., 2013; Soundappan et al., 2015). Thus far, a dichotomy has been proposed with SMAX1 (SUPPRESSOR OF MAX2-1) suppressing karrikin-related $\max 2$ phenotypes (e.g., germination and hypocotyl elongation) while other members of the SMXL family, namely SMXL6, SMXL7 and SMXL8, suppress SL-related phenotypes (e.g., shoot branching and lateral root density, Waters et al., 2017). While some specificity of SL or KAR/KL signalling is established through the receptors, additional specificity is reinforced through the degradation targets. And, these have been described not merely as suppressors of signalling but also as growth regulators, the activities of which are modulated through SL or KAR/KL signalling (Jiang et al., 2013). 
Here a role for KAI2-dependent and MAX2-dependent signalling in regulating specific root growth patterns (skewing, and waving) is demonstrated for the first time, which challenges the current model of SL/KL specificity with regards to their interacting partners from the SMXLs family. $\mathrm{KAR}_{2}$ or GR24 $4_{\text {rac }}$ (a synthetic analogue for SL and KAR) are shown to be poor analogues for KLs with regards to root skewing regulation. We propose that KAI2 and MAX2 regulate these growth patterns through a mechanism involving root radial expansion as the mutants' gravitropic and mechano-sensing responses remained largely unaffected. In addition, this work establishes new connections between MAX2 and SKS3 (SKU5 Similar), as we show genetic data placing both protein in the same genetic pathway regulating root skewing.

\section{Materials and Methods}

\section{Plant material and growth conditions}

Wild type Arabidopsis seeds Columbia-0 (Col) and Landsberg erecta (Ler) were the parental backgrounds for the mutants tested. Seeds for max2 (max2-1) and Atd14 (Atd14-1, (Waters et al., 2012) were provided by Prof. Dame Ottoline Leyser (SLCU, Stimberg et al., 2002). Seeds for $\max 2-7, \max 2-8$, kai2-1, kai2-2, dlk2-1, dlk2-2, dlk2-3, and KAI2:KAI2 (kai2-2) were a gift from Dr. Mark Waters (University of Western Australia, Waters et al., 2012; 2015b). Seeds were surface sterilized by treatment with $70 \%(\mathrm{v} / \mathrm{v})$ ethanol, followed by a rinse with sterile distilled water then incubation in $10 \%(\mathrm{v} / \mathrm{v})$ sodium hypochlorite, $0.05 \%(\mathrm{v} / \mathrm{v})$ Triton X-100 for 5 minutes at $20^{\circ} \mathrm{C}$ with shaking $(1,250 \mathrm{rpm})$. After a further five washes with sterile distilled water, seeds were placed on the surface of $0.8 \%(\mathrm{w} / \mathrm{v})$ agar (BD, UK) supplemented with $1 / 2$ MS (Murashige and Skoog including vitamins, $\mathrm{pH}$ 5.6, Duchefa, The Netherlands). Arabidopsis seeds were stratified in the dark for 2 days at $4{ }^{\circ} \mathrm{C}$, before transfer to a growth cabinet under controlled conditions at $23^{\circ} \mathrm{C}, 16 \mathrm{~h}$ light: $8 \mathrm{~h}$ dark, and $80 \mu \mathrm{mol} \mathrm{m} \mathrm{s}^{-2}$ irradiance. Growth plates were vertical unless stated otherwise.

\section{Root skewing assay}

After 9 days, images were taken by scanning plates from the back (i.e., roots were imaged through the agar) using a flat-bed scanner (300 dpi) and root skewing angles were measured in ImageJ (Schneider et al., 2012) using the angle tool. NeuronJ (Meijering et al., 2004) was used to record the $x$ and $y$ coordinates of the root tips and a marked section of the root. These coordinates were then used to calculate the horizontal growth index (HGI) and vertical growth index (VGI) as previously described (Grabov et al., 2004; Vaughn \& Masson, 2011). Waviness 
121

122

123

124

125

126

127

128

129

130

131

132

133

134

135

136

137

138

139

140

141

142

143

144

145

146

147

148

149

150

151

152

153

was measured as the ratio of the cord to the root length (Grabov et al., 2004; Vaughn \& Masson, 2011).

$G R 24_{\text {rac }}$ and $\mathrm{KAR}_{2}$

Plants were grown for 6 days on the surface of control medium $(0.8 \%(\mathrm{w} / \mathrm{v})$ agar supplemented with $1 / 2 \mathrm{MS}$, including vitamins, $\mathrm{pH} 5.6$ ), then transferred to medium containing racemic GR24 $4_{\text {rac }}$ (LeadGen Labs, USA) or $\mathrm{KAR}_{2}$ (Toronto Research Chemicals, Canada) or only the carrier for the test compound as a control (sterile distilled water for $\mathrm{KAR}_{2}$ and $0.02 \%(\mathrm{v} / \mathrm{v})$ acetone for GR24 $4_{\text {rac }}$. Plants were then grown for a further three days before scanning.

\section{Cell file rotation and root diameter analysis}

Images of the root tips from plants grown vertically for 6 days, then placed at a $45^{\circ}$ angle from the vertical for a further 3 days, were taken using a Leica DFC365FX camera attached to a Leica M205FA Stereo microscope (Leica Microsystems Ltd, UK) with a Planapo x1.6 objective set to magnification of $\mathrm{x} 80.5$. Images were stitched using the LAS X software platform (Leica Microsystems Ltd, UK). Following Wang et al. (Wang et al., 2011) cell file rotation (CFR) was defined as the number of epidermal cell files that crossed a $1 \mathrm{~mm}$ long straight line drawn down the longitudinal axis of the root from 1.5 to $2.5 \mathrm{~mm}$ from the root apex. Using the same images as for CFR measurements, root diameter was measured approximately $2 \mathrm{~mm}$ from the root apex using ImageJ (Schneider et al., 2012), three measurements were done per individual root.

\section{Mechanical stimulation assays for transcriptional response}

Plants were grown vertically on the surface of control plates for 9 days were transferred to a sterile buffer solution $\left(0.1 \mathrm{mM} \mathrm{KCl}, 10 \mathrm{mM} \mathrm{CaCl}_{2}\right.$ and $2 \mathrm{mM}$ bis-Tris propane, $\mathrm{pH} 5.8$ adjusted with 0.5 M MES). A total of 30 to 40 seedlings per genotype were transferred into a Petri dish ( $3 \mathrm{~cm}$ in diameter), containing $3 \mathrm{~mL}$ of buffer solution, and left to acclimatize on the bench for 3 hours with additional light (15W/865 Lumilux Daylight, maximum intensity: $86 \mu \mathrm{mol} \mathrm{m} \mathrm{s}^{-2}$ $\left.{ }^{1}\right)$. Mechanical stimulation was applied by shaking vigorously for 30 seconds, while control plants remained on the bench. Plants were then left untouched for a further 30 minutes after stimulation before being immersed in RNALater (Sigma Aldrich) for sample collection as described previously. For both assays, RNA was extracted from roots using the RNeasy Plant Mini kit (Qiagen) per manufacturer's instructions, including an additional DNase digestion 
step. A LiCl precipitation step was used to purify and concentrate the RNA before downstream qPCR analysis.

\section{cDNA synthesis and transcript abundance measurement}

Complementary DNA (cDNA) was synthesized from 500 ng RNA using the RT QuantiTect reverse transcription kit (Qiagen), following manufacturer's instructions except that incubation time was lengthened for the gDNA Wipeout step ( 3 minutes at $42^{\circ} \mathrm{C}$ ) and the cDNA synthesis (25 minutes at $42^{\circ} \mathrm{C}$ ). cDNA was used as template in a quantitative real-time PCR using the SYBR GREEN PCR kit (Qiagen) and the Rotor-Gene 3000 thermocycler (Qiagen) to determine transcript abundance of the genes of interest Calmodulin-like (CML) 12 and CML24. qPCR amplification cycle consisted of $5 \mathrm{~min}$ at $95^{\circ} \mathrm{C}$ followed by 40 cycles of $5 \mathrm{~s}$ at $95^{\circ} \mathrm{C}$ and $10 \mathrm{~s}$ at $60^{\circ} \mathrm{C}$. Melting curves (ramping from $55^{\circ} \mathrm{C}$ to $95^{\circ} \mathrm{C}$ rising $1{ }^{\circ} \mathrm{C}$ each step, with a $5 \mathrm{~s}$ delay between steps) were checked for unspecific amplification. qPCR traces were analysed using the $\mathrm{R}$ qpcR package (relevant parameters: data were normalized and the background subtracted; starting fit model: 14; efficiency estimation: cpD2; refmean: True; baseline subtraction using the average of the first 5 cycles; (Ritz \& Spiess, 2008) R package version 1.4-0. 2015) to calculate $\mathrm{Ct}$ values. Efficiencies (all $>92 \%$ ) were calculated using the calibration curve method. For each gene, the expression was calculated following the formula $\mathrm{E}=\left(\mathrm{eff}^{\mathrm{Ct}}\right)$. Expression of the genes of interest was normalised against two housekeeping genes Ubiquitin 10 (UBQ10) and Tubulin 4 (TUB4), as followed $\mathrm{R}_{\mathrm{Gene}}$ of Interest $=\mathrm{E}_{\mathrm{Gene}}$ of Interest $/\left(\operatorname{sqrt}\left(\mathrm{E}_{\mathrm{UBQ} 10} * \mathrm{E}_{\mathrm{TUB} 4}\right)\right)$. qPCR primers are listed in Table $\mathrm{S} 1$.

Measurements of cytosolic $\mathrm{Ca}^{2+}$ concentration $\left(\left[\mathrm{Ca}^{2+}\right]_{\text {cyt }}\right)$ in response to mechanical stimulation

Col and $\max 2$ (transformed using floral dip with Agrobacterium tumefaciens to express (apo)aequorin under a 35S promoter, Dodd et al., 2006)) were used at T3 or T4 generation to determine cytosolic free $\mathrm{Ca}^{2+}$ concentration $\left(\left[\mathrm{Ca}^{2+}\right]_{\mathrm{cyt}}\right)$. Equivalence of aequorin levels were determined by discharge assay of luminescence ( $>4$ million luminescence counts for both Col and $\max 2)$. Plants were grown vertically on solid medium for 7-8 days as described above. Excised root tips $(1 \mathrm{~cm}$ ) were placed in the wells (one root per well) of a white 96-well plate (Greiner Bio-One, UK) and incubated in $100 \mu \mathrm{L}$ of bathing solution $(10 \mu \mathrm{M}$ coelentrazine, Lux Biotechnology, UK), $0.1 \mathrm{mM} \mathrm{KCl}, 10 \mathrm{mM} \mathrm{CaCl}_{2}$ and $2 \mathrm{mM}$ bis-Tris propane, $\mathrm{pH} 5.8$ adjusted with $0.5 \mathrm{M} \mathrm{MES}$ ) for $2 \mathrm{~h}$ in the dark, at room temperature. Luminescence was then recorded 
187

188

189

190

191

192

193

194

195

196

197

198

199

200

201

202

203

204

205

206

207

208

209

210

211

212

213

214

215

216

217

218

219

every second in a plate-reading luminometer (FLUOstar Optima, BMG labtech, Ortenberg, Germany). After $35 \mathrm{~s}, 100 \mu \mathrm{L}$ of bathing solution (without coelentrazine) was injected into the well at $200 \mu \mathrm{L} \mathrm{s}^{-1}$ to cause a mechanical stimulus to the root resulting in a sudden increase in luminescence ("touch response"). The signal was monitored for a further $120 \mathrm{~s}$, when a $100 \mu \mathrm{L}$ of discharge solution ( $3 \mathrm{M} \mathrm{CaCl}_{2}$, in $30 \%(\mathrm{v} / \mathrm{v})$ ethanol) was delivered to normalize the luminescence data and calculate $\left[\mathrm{Ca}^{2+}\right]_{\mathrm{cyt}}\left(\right.$ Laohavisit et al., 2012). The $\left[\mathrm{Ca}^{2+}\right]_{\mathrm{cyt}}$ touch response of Col and $\max 2$ were then compared.

\section{Root gravitropism assays}

Arabidopsis plants were grown vertically for 14 days on the surface of control medium. On the day of the experiment, roots were positioned by aligning their root tips so that they could be imaged together. Plates were then placed vertically in the growth incubator but rotated through a $90^{\circ}$ angle thus inducing a $90^{\circ}$ change in gravitropic orientation. Root tips were imaged using a Raspberry Pi camera module (http://www.raspberrypi.org/). Images were acquired every 10 min for $10 \mathrm{~h}$. Image analysis was conducted using ARTT (Russino et al., 2013) which tracked the root tip growth and gave the tip orientation and displacement as output. Tip orientation was normalised to the displacement to take into account differences in growth rate.

\section{Data representation and statistical analysis}

Root skewing data were represented using beanplots constructed in the $\mathrm{R}$ environment (R Core Team, 2012) using the beanplot package (Kampstra, 2014), to show the variability in root skewing angle. Statistical analyses were also conducted in the $\mathrm{R}$ environment. Normal distribution of the data and equality of variance were verified using Shapiro and Levene tests (Lawstat package, Gastwirth et al., 2017), respectively. Significant differences amongst genotypes were verified using one-way Analysis of Variance (ANOVA), followed by Tukey HSD. ANOVAs were conducted on rank values as a non-parametric method, when data did not uphold the assumptions of normality and homoscedasticity. All experiments were repeated at least three times.

\section{Results}

\section{Mutation in kai2 and $\max 2$ increases root rightward skew}

If $\mathrm{KL}$ or karrikins were involved in root skewing then insensitive mutants of Arabidopsis thaliana would display an aberrant root-skewing phenotype. Vertically-grown kai2-1 and kai2- 
2 mutants showed significantly increased rightward root skewing compared to the Ler wild type ( $\alpha$, root tip displacement, viewed from the back of the plate: Fig. 1a, b; Tukey HSD, $p<$ $0.01)$. Vertically-grown $\max 2-7$ and $\max 2-8$ mutants also showed a significant increase in rightward root skewing compared to wild type (Fig. 1a, b; Tukey HSD, $p<0.01$ ).

Horizontal Growth Index (HGI; ratio of root tip displacement along the $x$ axis to root length, Grabov et al., 2004; Vaughn \& Masson, 2011) was also significantly higher in kai2-1, kai2-2, $\max 2-7$, and $\max 2-8$ compared to wild type (Fig. 1c; Tukey HSD, $p<0.01$ ), supporting the skewing angle data and showing increased deviation from vertical by mutant roots. Similarly, the Vertical Growth Index (VGI; ratio of root tip displacement along the $y$ axis to root length, Grabov et al., 2004; Vaughn \& Masson, 2011) was significantly smaller for kai21, kai2-2, $\max 2-7$, and $\max 2-8$ compared to wild type (Fig. 1b; Tukey HSD, $p<0.01$ ). In separate experiments, two complemented kai2-2 lines (driven by the native promoter KAI2:KAI2 (kai2-2), Waters et al., 2015b) showed significantly decreased root skewing angle compared to kai2-2 (Fig. S1a; Tukey HSD, $p<0.05$ ). Overall these data suggest a role for both KAI2 and MAX2 in root skewing.

\section{$K A I 2$ and $M A X 2$ operate through the same genetic pathway}

Although no physical interaction has been demonstrated between KAI2 and MAX2, they have been placed in the same signalling pathway through genetic studies of elongated hypocotyl phenotypes (Waters et al., 2012). Here, the double mutant kai2-2 max2-8 showed a significantly increased rightward root skewing compared to wild type (Fig. 2a, b, Tukey HSD, $p<0.05$ ), which was not significantly different from that of kai2-2 (Fig. 2a, b; Tukey HSD, n.s.). That skewing angle of the kai2-2 $\max 2-8$ double mutant was not greater than that of the kai2-2 single mutant suggests that KAI2 and MAX2 operate in the same pathway. Critically, $d 14$ mutants that are insensitive to SL but not KAR (Waters et al., 2012) showed no significant increase in root skewing compared to wild type (Fig. 2c, d, ANOVA, n.s.). A higher root skewing angle for wild type Ler plants compared to Col was found, as noted previously (Vaughn \& Masson, 2011). Moreover, the root skewing of dlk2 mutants (Waters et al., 2012) was not significantly different to wild type (Fig. S1b; Tukey HSD, n.s.). As the DLK2 protein is related to both KAI2 and D14, overall these data demonstrate a specific role for KAI2 and MAX2 in the regulation of root skewing and thus implicate KL/KAR sensing through these proteins. 


\section{$\mathrm{KAR}_{2}$ reduces root skewing}

In the absence of purified and identified KL compounds, the effect of KAR on root skewing was tested using the potent karrikin $\mathrm{KAR}_{2}$ (Nelson et al., 2009; Waters et al., 2015a). The phenotype of kai2 and $\max 2$ mutants suggests that an impairment in KL perception leads to greater rightward root skewing. Therefore, an increased availability of KL or its analogue

259

260

261

262

263

$\mathrm{KAR}_{2}$ might compensate for a lowered sensitivity of the system and decrease the rightward root skewing. Here there was a significant effect of $\mathrm{KAR}_{2}$ in reducing rightward root skewing of Ler wild type plants with concentrations of 5 and $10 \mu \mathrm{M}$ (Fig. S2a Tukey HSD, $p<0.05$ ). A significant inhibitory effect on primary root elongation of Ler plants was evident at $10 \mu \mathrm{M}$ $\mathrm{KAR}_{2}$ (Fig. S2b, Tukey HSD, $p<0.01$ ).

The presence of 2.5 and $5 \mu \mathrm{M} \mathrm{KAR}_{2}$ in the medium also significantly decreased the root skewing angle of kai2-2 (Fig. S2b; Tukey HSD, $p<0.05$ ). kai2 plants seem to be more sensitive to $\mathrm{KAR}_{2}$ than Ler plants. The KAI2-independent effect of $\mathrm{KAR}_{2}$ on root skewing may also be linked to reduced root elongation, as this was significantly lower in the presence of $5 \mu \mathrm{M} \mathrm{KAR}_{2}$ (Fig. S2b, Tukey HSD, $p<0.01$ ) but not at $2.5 \mu \mathrm{M}$ (Fig. S2b, Tukey HSD, n.s.). It is likely that kai2 plants are more sensitive to the unspecific toxicity effect of $\mathrm{KAR}_{2}$ than Ler because they lack a mechanism for degradation of KAR and KL. Similarly, the presence of $5 \mu \mathrm{M} \mathrm{KAR}_{2}$ in the medium significantly decreased the root skewing angle of $\max 2-$ 8 (Fig. S2e; Tukey HSD, $p<0.01$ ) as well as primary root elongation (Fig. S2f; Tukey HSD, $p<0.01)$.

\section{GR24 $_{\text {rac }}$ has little effect on root skewing}

Because of the structural similarities between KAR and the SL analogue GR24 $4_{\text {rac }}$ (Zwanenburg et al., 2009), and the already established role of GR24 $4_{\text {rac }}$ in regulating root growth (RuyterSpira et al., 2011; Kapulnik et al., 2011; Rasmussen et al., 2012), we tested the effect of GR24 rac on root skewing. A racemic mix of GR24 (GR24 rac) that can also be perceived by KAI2 (Scaffidi et al., 2014; Waters et al., 2015a) was tested at 1 and $5 \mu \mathrm{M}$ as greater concentrations tend to have a toxicity effect on root growth (Ruyter-Spira et al., 2011). Treatment with GR24 $4_{\text {rac }}$ led to a small increase in rightward root skewing in Ler plants at $1 \mu \mathrm{M}$ (Fig. S3a, Tukey HSD, $p<0.01$ ) but not at $5 \mu \mathrm{M}$ GR24 $4_{\text {rac }}$ (Tukey HSD, n.s.). There was no significant effect of 1 or $5 \mu \mathrm{M}$ GR24 $4_{\text {rac }}$ on kai2-1 root skewing (Tukey HSD, n.s.). There was no significant effect of $1 \mu \mathrm{M}$ GR2 $4_{\text {rac }}$ on root skewing of kai2-2 (Tukey HSD, n.s.) and there 
was a small but significant decrease in kai2-2 root skewing with $5 \mu \mathrm{M}$ GR24 rac (Tukey HSD, $p<0.01)$. There was no significant effect of 1 or $5 \mu \mathrm{M}$ GR2 $4_{\text {rac }}$ on the root skewing of Col plants (Fig. S3b, ANOVA, $\mathrm{F}_{(2,261)}=1.26$, n.s.). There was a small but significant increase in root skewing angle for $\max 2-1$ plants in the presence of $1 \mu \mathrm{M}$ GR2 $4_{\text {rac }}$ (Tukey HSD, $p<0.05$ ) but not $5 \mu \mathrm{M} \mathrm{GR24}$ rac (Tukey HSD, n.s.), while $d 14$ plants did not respond to the presence of GR24 $4_{\text {rac }}$ (ANOVA, $F_{(2,184)}=1.31$, n.s.). Overall, GR24 $4_{\text {rac }}$ had little effect on root skewing especially in comparison with the root skewing angles of kai2 and max 2 mutants, and as such is a poor $\mathrm{KL}$ analogue with regards to the regulation of root skewing.

\section{MAX2 regulation of skewing operates through SMXL6,7,8 but not SMAX1}

The involvement of MAX2 degradation targets, SMAX1 (SUPPRESSOR OF MAX2-1) and SMXL (SUPPRESSOR OF MAX2-1-LIKE, (Stanga et al., 2013) in regulating root skewing was examined. D53, a homologue of SMXL6,7,8 in rice, forms a complex with D14 and D3, and is degraded following SL treatment (Jiang et al., 2013; Zhou et al., 2013). The current mechanistic model for Arabidopsis is that SMAX1 is important for the KL part of the signalling pathway whereas SMXL6,7,8 are more relevant to the SL part of the pathway (Soundappan et al., 2015).

Here we tested the hypothesis that the loss of SMAX1 or SMXL6,7,8 proteins would affect root skewing. Both smaxl-2 and $\operatorname{smxl6,7,8}$ mutants had a significantly decreased skew compared to wild type (Fig. 3a, b; Tukey HSD, $p<0.01$ ), thus suggesting that the abundance of these proteins is important in regulating the skew. The hypothesis that the MAX2-dependent regulation of SMAX1 and SMXL6,7,8 protein abundance is relevant to the root skewing phenotype was then tested. For this, the root skewing angle of the max $2 \operatorname{smax} 1-2$ double mutant as well as the quadruple mutant $\max 2-1 \operatorname{smxl6,7,8}$ was measured. If MAX2 were to affect root skewing exclusively through the abundance of SMAX1 or SMXL6,7,8, then the presence of the $\max 2$ mutation should have no effect on the root skewing phenotype of smax1-2 or smxl6,7,8. Here, a significant increase in root skewing angle in the smax1-2 max2-1 double mutant compared to smaxl (Tukey HSD, $p<0.01$ ) was observed, but there was no further

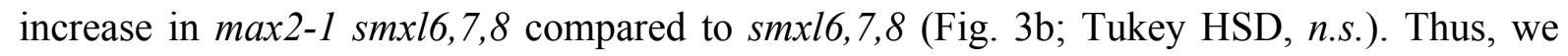
conclude that the regulation of root skewing by MAX2 is dependent on SMXL6,7,8 rather than SMAX1.

\section{KAI2 and MAX2 negatively regulates both skewing on a tilted surface and waving}


319

320

321

322

323

324

325

326

327

328

329

330

331

332

333

334

335

336

337

338

339

340

341

342

343

344

345

346

347

348

349

350

351

Positioning plates at a $45^{\circ}$ angle rather than $90^{\circ}$ increases root skewing angle. A significant increase in rightward root skew angle was observed here for the Ler wild type grown at a $45^{\circ}$ plate angle (Fig. 4a, b, ANOVA, $\mathrm{F}_{(1,510)}=134.9$, p <0.001), whilst kai2-1, kai2-2, max2-7, and max 2-8 also showed a significantly increased rightward root skewing angle compared to Ler (Fig. 3a,b; Tukey HSD, $p<0.01$ ). The increase in mutant root skew relative to wild type was maintained at the $45^{\circ}$ plate angle compared to growth at $90^{\circ}$, indicating that loss of KAI 2 or MAX2 did affect the mutant's ability to sense and respond to the tilt.

Although mechanistic models for root skewing vary (Roy \& Bassham, 2014), the rotation of epidermal cell files is considered to be an important feature (Sedbrook et al., 2002; Oliva \& Dunand, 2007; Wang et al., 2011). Right-handed cell file rotation was significantly increased in both kai2-2 (mean \pm SEM $6.93 \pm 0.44$ cell mm $^{-1}$; Tukey HSD, $p<0.01$ ) and $\max 2$ $8\left(5.13 \pm 0.30\right.$ cell mm${ }^{-1}$; Tukey HSD, $\left.p=0.08\right)$ compared to Ler wild type $(4.24 \pm 0.25$ cell $\mathrm{mm}^{-1}$; Fig. 3c).

Increased root skewing is often also accompanied by increased root waving (Roy \& Bassham, 2014) - a decrease in root straightness calculated as the ratio of the cord over the root length (i.e., straight roots have a ratio of 1 and the lower the ratio the less straight/more wavy the root; Grabov et al., 2004; Vaughn \& Masson, 2011). Growth on a tilted surface can also decrease straightness (Roy \& Bassham, 2014). When grown at $90^{\circ}$ plate angle, both kai2-1 and kai2-2 showed a significantly decreased straightness compared to Ler wild type (Fig. 4d; Tukey HSD, $p<0.05$ ) and similarly when grown at $45^{\circ}$ (Fig. 4d; Tukey HSD, $p<0.01$ ). Ler was significantly less straight at $45^{\circ}$ compared to $90^{\circ}$ (Tukey HSD, $p<0.01$ ). These data show that KAI2 is involved in the negative control of both skewing and waving when plants are grown vertically and at an angle.

\section{kai2 and $\max 2$ can support a normal mechano-sensing transcriptional response}

The growth responses of the kai2 mutants on tilted plates suggested that the mutation does not affect the root tip's ability to sense the increased mechanical impedance afforded by the inclined growth medium. Rather, that the kai2 mutants have an exaggerated root skew when grown on a tilted surface suggests that downstream responses are impaired. To test for a role for KAI2 in mechano-sensing responses seedlings were subjected to mechanical stress prior to determination of root transcript levels of CML12 and CML24 (CALMODULIN LIKE PROTEIN, Fig. 5a). These transcripts are known to increase upon mechanical stimulation (Braam \& Davis, 1990). These tests also addressed max2 and $d 14$ in the Col background (Fig. 
5b). Mechanical stimulation caused significant upregulation of CML12 and CML24 transcript in roots of all genotypes tested (ANOVA, $p<0.001$ ) but no mutants responded significantly differently to their wild type. Thus, the data suggest that root transcriptional mechanoresponsiveness is not drastically altered in either KL- or SL-insensitive mutants.

As a final test for alteration in mechano-sensing and response, max2 (as the common lesion in KL- and SL-pathways) was transformed to express (apo)aequorin as a reporter of cytosolic free $\mathrm{Ca}^{2+}\left(\left[\mathrm{Ca}^{2+}\right]_{\mathrm{cyt}}\right) .\left[\mathrm{Ca}^{2+}\right]_{\text {cyt }}$ increases transiently in response to mechano stimulation, acting as a second messenger (Knight et al., 1991). There was no significant difference between baseline level pre-injection and post-injection for Col (t-test, n.s.) or max2 (t-test, n.s.). There was no significant difference in the amplitude of the touch-induced peak increase in $\left[\mathrm{Ca}^{2+}\right]_{\text {cyt }}$ between genotypes (Fig. 6 ; $t$-test, n.s.). However, the total $\mathrm{Ca}^{2+}$ mobilised over the recording period (excluding the discharge) for $\max 2(33.99 \mu \mathrm{Ms} \pm 0.57)$ was significantly higher than that for $\operatorname{Col}(29.91 \mu \mathrm{Ms} \pm 0.49 ; t$-test, $\mathrm{p}<0.01)$.

\section{kai2 has a slower early gravitropic response}

Agravitropic mutants can also show an increased root skewing (Okada \& Shimura, 1990). To investigate whether an aberrant gravitropic response of kai2-2 plants contributed to their skewing phenotype, root tip orientation was monitored every $10 \mathrm{~min}$ after gravistimulation for $10 \mathrm{~h}$. Both kai2-2 and wild type responded significantly with a change of tip orientation over time (Fig. 7; ANOVA $\mathrm{F}_{(1,4022)}=46.8, p<0.001$ ). Comparisons of the responses (normalised for elongation rate) using ANOVA showed that there was a significant interaction between time and genotype (ANOVA, $\mathrm{F}_{(1,4022)}=40.9, p<0.01$ ), indicating a difference in gravitropic response between genotypes. kai2-2 root tip angle started to decrease later than Ler. After 100 min, the angle of kai2-2 was significantly higher than that of Ler (ANOVA, $\mathrm{F}_{(1,64)}=4.4, p<$ 0.01 ) but at $600 \mathrm{~min}$ there was no significant difference (ANOVA, $\mathrm{F}_{(1,64)}=0.24$, n.s.). Overall, the difference in gravitropic response between kai2-2 and Ler may be a small contributory factor to root skewing, but occurring only in the early stages of the response.

\section{MAX2 regulation of root skewing involves SKS3 and SKU5}

Similarly to the kai2 and max2 mutants, mutant plants deficient in the SKU5 protein that is linked to the plasma membrane by a glycosylphosphatidylinositol (GPI) anchor also showed an increased rightward root skewing phenotype, increased CFR with no change in gravitropic 
385

386

387

388

389

390

391

392

393

394

395

396

397

398

399

400

401

402

403

404

405

406

407

408

409

410

411

412

413

414

415

416

417

response (Sedbrook et al., 2002). In our experiments, sku5 also displayed a rightward skew when grown vertically that was significantly greater than the wild type (Fig 8b, Tukey HSD, $p$ $<0.05$ ). There was no further increase in $s k u 5 \max 2$ compared to $\max 2$ (Tukey HSD, n.s.), showing that SKU5 and MAX2 can regulate root skewing in the same pathway but as the skewing angle of the sku 5 max 2 mutant was significantly higher than that of sku 5 (Tukey HSD, $p<0.001)$ this suggests that part of the MAX2 pathway is SKU5-independent. The sks3 (sku5 similar 3) mutant deficient in a SKU5-related protein also showed a decreased rightward root skewing (Tukey HSD, $p<0.05$ ) that was maintained even in the absence of MAX2 (comparison sks3: sks3 max2-1, Tukey HSD, n.s.). These data suggest that the abundance of SKS3 protein may itself regulate root skewing and that the abundance of this protein may be regulated through the MAX2 pathway. sks3 and sku5 do suppress the high LRD of $\max 2$ mutants (Fig. S4) as well as the decreased germination rate (Fig. S5). Thus, our data suggest that members of the SKU/SKS at least SKS3 are degradation targets for the MAX2 pathway, and in the case of SKS3 specifically regulating of root skewing. The genetic link established here between MAX2 and SKU/SKS family points towards a role of MAX2 in regulating, through SKS3, a cell wall-dependent process.

\section{KAI2 and MAX2 positively regulate root diameter}

Given the subtle responses in terms of gravitropism and mechanical stimulation versus the clear increase in CFR and link with members of the SKU/SKS family, we hypothesise that in both the kai2 and $\max 2$ mutants the root skewing phenotype arises due to a restriction of cell growth. This is supported by our finding that the mean root diameter of the mutants was significantly narrower than that of wild type (Fig. S6, Ler $166.43 \mu \mathrm{m} \pm 1.79$, kai2-2 $155.57 \mu \mathrm{m} \pm 1.41$, $\max 2-8146.59 \mu \mathrm{m} \pm 1.67$; Tukey HSD $p<0.001$ ), suggesting that root radial expansion may be restricted.

\section{Discussion}

Evidence here demonstrating a role for KAI2 and MAX2 in regulating root skewing and waving in Arabidopsis reinforces the idea that plant endogenous KL can act as a phytohormone (Conn \& Nelson, 2016). This is the first root growth phenotype characterised for karrikininsensitive mutants in a non-host species (Gutjahr et al. 2015).

\section{KAI2 and MAX2 as new regulatory components for root skewing}


418 The characterization of different root skewing and waving abilities amongst Arabidopsis

419 ecotypes strongly suggests that the surface-dependent growth patterns represent an adaptive 420 response relevant to natural soil conditions (Vaughn \& Masson, 2011; Schultz et al., 2017).

421 Mutants have proved useful in identifying new components of the machinery regulating root

422 skewing in Arabidopsis. Here the increased root skewing phenotype of kai2 and max2 suggests

423 that both KAI2 and MAX2 negatively regulate root skewing. Since these two proteins are

424 involved in the perception of KAR and KL this provides evidence supporting a role for KL in

425 regulating root skewing. Previous studies have shown an involvement of the SL pathway in the

426 regulation of root system architecture (although skewing and waving were not reported)

427 (Ruyter-Spira et al., 2011; Kapulnik et al., 2011; Rasmussen et al., 2012). We found no

428 evidence supporting a role for endogenous SL in root skewing, since the $d 14$ mutant impaired

429 in the perception of SL does not show a root skewing phenotype.

430 For phenotypes such as elongated hypocotyls or increased seed dormancy (Waters et 431 al., 2012), $\mathrm{KAR}_{2}$ acts as a good synthetic analogue for KL (Conn \& Nelson, 2016). However, this is not the case for root skewing, in which a high concentration of $\mathrm{KAR}_{2}$ is necessary to induce a phenotype and is KAI2 independent. A similarly high $(10 \mu \mathrm{M})$ concentration of the less potent $\mathrm{KAR}_{1}$ could also induce a KAI2-dependent reduction in hypocotyl length (Waters et al., 2012). $\mathrm{KAR}_{1}$ is also less potent than $\mathrm{KAR}_{2}$ in targeting the degradation of KAI2 protein (Waters et al., 2015a). Results here suggest that KL represent a family of related compounds that can regulate different aspects of plant development, and that the KL responsible for regulating root skewing may differ from the KL responsible for regulating hypocotyl elongation and seed germination. Many SL compounds have been purified thus far (Bouwmeester et al., 2007), perhaps structural diversity is also the case for KL. Although $\mathrm{KAR}_{2}$ can regulate root skewing, the high concentration required plus the independence from $\mathrm{KAI} 2$ and MAX2 suggest that $\mathrm{KAR}_{2}$ is likely non-specific and does not represent a good analogue of KL.

\section{Root skewing phenotype suggests new links between MAX2 and SKS proteins}

The mechanism by which KAI2 and MAX2 regulates root skewing remains elusive but must involve a differential growth leading to increased epidermal cell file rotation. We found no evidence supporting a role for KAI2 and MAX2 in regulating the root mechano-sensing transcriptional response and only a very subtle effect of MAX2 on mechano-stimulated $\left[\mathrm{Ca}^{2+}\right]_{\text {cyt }}$ response. The link established here between MAX2 and SKU5 as well as MAX2 and 
modification or integrity. Amongst the 11 highly probable skew gene candidates identified in Arabidopsis roots using microarrays, three were associated to the cell wall either because of their physical location (PAP24), or because of their role in cell wall integrity (DIN2) or formation (MIOX4, Schultz et al., 2017). SKS15 also presented an expression pattern indicative of a possible role in root skewing in this study. However, analysis of the cell wall composition using Fourier transform infrared spectroscopy and analysis of neutral sugars revealed no differences between sku5 mutant and wild type (Sedbrook et al., 2002).

Several lines of evidence suggest that KL and KAR affect cell wall composition. First, amongst the 133 genes that are differentially regulated $24 \mathrm{~h}$ post-imbibition with $1 \mu \mathrm{M} \mathrm{KAR}{ }_{1}$, 11 relate to cell wall (Nelson et al., 2010). Genes annotated as being part of the 'plant-cell type cell wall' category of the GO cellular component were significantly enriched in the set of genes regulated by $\mathrm{KAR}_{1}$. Amongst those genes, sks17 (SKU5 similar 17) was found to be upregulated 2.2-fold upon treatment with $\mathrm{KAR}_{1}$. It is unclear whether the levels of proteins also increase upon treatment with $\mathrm{KAR}_{1}$. Second, metabolomic analyses showed reduced levels of flavonoids contributing to lignin composition (including $\rho$-coumaric acids and ferulic acids) in $\max 2$ roots compared to wild type roots under control conditions (Walton et al., 2016). These are also good indicators of lower levels of cutin monomer, which signals in the AM-root symbiosis (Wang et al. 2012). Thus, an altered cell wall would fit with the impairment in the early events leading to the establishment of KAI2-dependent AM symbiosis in host species (Gutjahr et al., 2015) and could feasibly influence root skewing and waving.

\section{Root skewing phenotype challenges the current model for the SMXLs}

Soundappan et al. (2015) suggested specific relationships between SMAX1 and KAI2-KAR/ KL-regulated growth and between SMXL6,7,8 and D14-SL- regulated growth. However, data here do not support the idea that there is a clear dichotomy in terms of the degradation-target proteins involved in the perception pathways for SL and KL. Rather the data support a role for MAX2 in regulating the skew in a D14-independent pathway through SMXL6,7,8 rather than SMAX1. However, this is complicated by the fact that SMAX1 itself appears to regulate root skewing. One explanation for this observation might be that SMAX1 regulates the skew indirectly via the regulation of SMX6,7,8. In this scenario, the smax 1-2 mutant has a skewing phenotype because of a decreased level of SMXL6,7,8, proteins. The lack of direct effect of SMAX1 on root skewing is also supported by the fact that there is no further increase in root

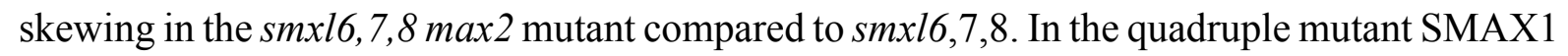


485

486

487

488

489

490

491

492

493

494

495

496

497

498

499

500

501

502

503

504

505

506

507

508

509

510

511

512

513

514

515

516

517

protein levels should be different because SMAX1 is regulated through MAX2 (Stanga et al., 2013; Soundappan et al., 2015). Similarly, the level of SMXL6,7,8 should be higher in the smax1-2 max2-1 compared to smax 1-2, thus leading to the observed increase in root skewing and supporting a role for SMXL6,7,8 in regulating root skewing.

In addition, a role was found for KAI2 and MAX2 but not D14 in regulating root skewing. Overall, this suggests that with regards to the regulation of root growth patterns, SMXL6,7,8 as well as SMAX1 may be involved in the MAX2-dependent regulation of skewing, which was also found to be KL-dependent rather than SL-dependent. Much may depend on the spatial localisation of proteins. SMAX1 is expressed in the root cap, while SMXL6, 7 and 8 are also present in the vasculature or mature roots (Soundappan et al., 2015). KAI2 expression could be found preferentially in the vasculature (Brady et al., 2007) potentially favouring interaction with SMXL6, 7 or 8.

\section{Conclusions}

Root positioning in the soil is critical in terms of regulating access to nutrients and water, but also interaction with neighbours (Fang et al., 2013). The regulation of root positioning is dependent on both the genetic and environmental response. While it is difficult to argue for the field-relevance of root skewing patterns observed on the surface of agar plates, the characterization of different root skewing and waving abilities amongst Arabidopsis ecotypes strongly suggests that the surface-dependent growth patterns represent an adaptive response relevant to natural soil conditions (Vaughn \& Masson, 2011). The involvement of both KAI2 and MAX2 suggests a role for a potential new phytohormone KL, in regulating root skewing and waving.

\section{Acknowledgments}

We thank Dr. Mark Waters and Prof. Dame Ottoline Leyser for providing seeds and commenting on the manuscript. Thank you also to Prof. David Nelson for providing seeds and Daniel Safka for support in setting up the raspberry Pi system. We are grateful to Dr. Uta Paszkowski, Prof. Alex Webb, Dr. Siobhan Braybrook, Prof. Sidney Shaw and Dr. Jenny Mortimer for interesting discussions. Work was supported by the Broodbank Trust, the Newton Trust, the Gatsby Foundation, and the BBSRC Doctoral Training Programme (BB/J014540/1)

Authors Contribution 
S.M.S. and J.M.D. planned and designed the research. S.M.S., Y.G., E.M. and F.J. performed experiments and analysed data. S.M.S. and J.M.D. wrote the manuscript.

\section{Conflict of interest}

The authors have no conflict of interest to declare.

Table S1: Primer sequences used in qPCR analysis.

\begin{tabular}{|l|l|l|}
\hline Gene & Direction & Sequence \\
\hline$C M L 12$ & Forward & 5'-AAGCCTTCCGCGTATTCGACAAGAA-3' \\
\hline$C M L 12$ & Reverse & 5'-CACAAACTCAGAGAAACTGATGGTTCC-3' \\
\hline$C M L 24$ & Forward & 5'-GAGTAATGGTGGTGGTGCTTGA-3' \\
\hline$C M L 24$ & Reverse & 5'-ACGAATCATCACCGTCGACTAA-3' \\
\hline$U B Q 10$ & Forward & 5'-CCGACTACAACATTCAGAAGGA-3' \\
\hline$U B Q 10$ & Reverse & 5'-TCAGAACTCTCCACCTCCAAA-3' \\
\hline$T U B 4$ & Forward & 5'-CTGTTTCCGTACCCTCAAGC-3' \\
\hline$T U B 4$ & Reverse & 5'-AGGGAAACGAAGACAGCAAG-3' \\
\hline
\end{tabular}

\section{References:}

Bouwmeester HJ, Roux C, Lopez-Raez JA, Bécard G. 2007. Rhizosphere communication of plants, parasitic plants and AM fungi. Trends in Plant Science 12: 224-230.

Braam J, Davis RW. 1990. Rain-, Wind-, and Touch-Induced Expression of Calmodulin and Calmodulin-Related Genes in Arabidopsis. Cell 60: 357-364.

Brady S, Orlando D, Lee J, Wang J, Koch J, Dinneny J, Mace D, Ohler U, Benfey P. 2007. A high-resolution root spatiotemporal map reveals dominant expression patterns. Science 318: 801-806.

Buer CS, Masle J, Wasteneys GO. 2000. Growth conditions modulate root-wave phenotypes in Arabidopsis. Plant Cell Physiology 41: 1164-1170.

Buer CS, Wasteneys GO, Masle J. 2003. Ethylene modulates root-wave responses in Arabidopsis. Plant Physiology 132: 1085-1096.

Bythell-Douglas R, Waters MT, Scaffidi A, Flematti GR, Smith SM, Bond CS. 2013. The structure of the karrikin-insensitive protein (KAI2) in Arabidopsis thaliana. PLoS ONE 8: e54758.

Chevalier F, Nieminen K, Sanchez-Ferrero JC, Rodriguez ML, Chagoyen M, Hardtke CS, Cubas P. 2014. Strigolactone promotes degradation of DWARF14, an $\alpha / \beta$ Hydrolase Essential for Strigolactone Signaling in Arabidopsis. The Plant Cell 26: 1134-1150. 
Conn CE, Nelson DC. 2016. Evidence that KARRIKIN-INSENSITIVE2 (KAI2) receptors may perceive an unknown signal that is not karrikin or strigolactone. Frontiers in Plant Science 6: 1219.

550

551

552

553

554

555

556

557

558

559

560

561

562

563

564

565

566

567

568

569

Darwin C, Darwin F. 1880. The power of movement in plants. New, York: D. Appleton and Company.

Dodd AN, Jakobsen MK, Baker AJ, Telzerow A, Hou S-W, Laplaze L, Barrot L, Scott Poethig R, Haseloff J, Webb AAR. 2006. Time of day modulates low-temperature $\mathrm{Ca}^{2+}$ signals in Arabidopsis. The Plant Journal 48: 962-973.

Fang S, Clark RT, Zheng Y, Iyer-Pascuzzi AS, Weitz JS, Kochian LV, Edelsbrunner H, Liao H, Benfey PN. 2013. Genotypic recognition and spatial responses by rice roots.

Proceedings of the National Academy of Sciences 110: 2670-2675.

Flematti GR, Dixon KW, Smith SM. 2015. What are karrikins and how were they 'discovered' by plants? BMC Biology 13: 108.

Gastwirth JL, Gel YR, Hui W, Lyubchich V, Miao W. 2017. Tools for Biostatistics, Public Policy and Law. R package version. 1-44.

Grabov A, Ashley MK, Rigas S, Hatzopoulos P, Dolan L, Vicente-Agullo F. 2004. Morphometric analysis of root shape. New Phytologist 165: 641-652.

Gutjahr C, Gobbato E, Choi J, Riemann M, Johnson MG, Summers W, Carbonnel S, Mansfield C, Yang S-Y, Nadal M, et al. 2015. Rice perception of symbiotic arbuscular mycorrhizal fungi requires the karrikin receptor complex. Science 350: 1516-1521.

Hamiaux C, Drummond RSM, Janssen BJ, Ledger SE, Cooney JM, Newcomb RD, Snowden KC. 2012. DAD2 is an alpha/beta; hydrolase likely to be involved in the perception of the plant branching hormone, strigolactone. Current Biology 22: 2032-2036.

Jiang L, Liu X, Xiong G, Liu H, Chen F, Wang L, Meng X, Liu G, Yu H, Yuan Y, et al. 2013. DWARF 53 acts as a repressor of strigolactone signalling in rice. Nature 504: 401405.

Kampstra P. 2014. Beanplot: A Boxplot Alternative for Visual Comparison of Distributions. Journal of Statistical Software, Code Snippets 28: 1-9.

Kapulnik Y, Delaux P-M, Resnick N, Mayzlish-Gati E, Wininger S, Bhattacharya C, Séjalon-Delmas N, Combier J-P, Bécard G, Belausov E, et al. 2011. Strigolactones affect lateral root formation and root-hair elongation in Arabidopsis. Planta 233: 209-216.

Knight M, Campbell AK, Smith SM, Trewavas A. 1991. Transgenic plant aequorin reports the effects of touch and cold-shock and elicitors on cytoplasmic calcium. Nature 352: 524526.

Kushwah S, Jones AM, Laxmi A. 2011. Cytokinin interplay with ethylene, auxin, and glucose signaling controls Arabidopsis seedling root directional growth. Plant Physiol 156: 1851-1866.

Lanza M, Garcia-Ponce B, Castrillo G, Catarecha P, Sauer M, Rodriguez-Serrano M, 
Páez-García A, Sánchez-Bermejo E, TC M, del Puerto YL, et al. 2012. Role of actin cytoskeleton in brassinosteroid signaling and in its integration with the auxin response in plants. Developmental Cell 22: 1275-1285.

\section{Laohavisit A, Shang Z, Rubio L, Cuin TA, Very AA, Wang A, Mortimer JC,} Macpherson N, Coxon KM, Battey NH, et al. 2012. Arabidopsis Annexin1 Mediates the Radical-Activated Plasma Membrane $\mathrm{Ca}^{2+}$ and $\mathrm{K}^{+}$-Permeable Conductance in Root Cells. The Plant Cell 24: 1522-1533.

Meijering E, Jacob M, C J, Sarria F, Steiner P, Hirling H, Unser M. 2004. Design and validation of a tool for neurite tracing and analysis in fluorescence microscopy Images. 1-14.

Nelson DC, Flematti GR, Riseborough JA, Ghisalberti EL, Dixon KW, Smith SM. 2010. Karrikins enhance light responses during germination and seedling development in Arabidopsis thaliana. Proceedings of the National Academy of Sciences 107: 7095-7100.

Nelson DC, Riseborough JA, Flematti GR, Stevens J, Ghisalberti EL, Dixon KW, Smith SM. 2009. Karrikins discovered in smoke trigger Arabidopsis seed germination by a mechanism requiring gibberellic acid synthesis and light. Plant Physiology 149: 863-873.

Okada K, Shimura Y. 1990. Reversible root tip rotation in Arabidopsis seedlings induced by obstacle-touching stimulus. Science 250: 274-276.

Oliva M, Dunand C. 2007. Waving and skewing: how gravity and the surface of growth media affect root development in Arabidopsis. New Phytologist 176: 37-43.

\section{Rasmussen A, Mason MG, De Cuyper C, Brewer PB, Herold S, Agusti J, Geelen D,} Greb T, Goormachtig S, Beeckman T, et al. 2012. Strigolactones suppress adventitious rooting in Arabidopsis and pea. Plant Physiology 158: 1976-1987.

Ritz C, Spiess AN. 2008. qpcR: an R package for sigmoidal model selection in quantitative real-time polymerase chain reaction analysis. Bioinformatics 24: 1549-1551.

Roy R, Bassham DC. 2014. Root growth movements: Waving and skewing. Plant Science 221-222: 42-47.

Russino A, Ascrizzi A, Popova L, Tonazzini A, Mancuso S, Mazzolai B. 2013. A novel tracking tool for the analysis of plant-root tip movements. Bioinspiration \& Biomimetics $\mathbf{8}$ : 025004-16.

Ruyter-Spira C, Kohlen W, Charnikhova T, Van Zeijl A, Van Bezouwen L, De Ruijter N, Cardoso C, Lopez-Raez JA, Matusova R, Bours R, et al. 2011. Physiological effects of the synthetic strigolactone analog GR24 on root system architecture in Arabidopsis: another belowground role for strigolactones? Plant Physiology 155: 721-734.

Scaffidi A, Waters MT, Sun YK, Skelton BW, Dixon KW, Ghisalberti EL, Flematti GR, Smith SM. 2014. Strigolactone hormones and their stereoisomers signal through two related receptor proteins to induce different physiological responses in Arabidopsis. Plant Physiology 165: 1221-1232.

Schneider CA, Rasband WS, Eliceiri KW. 2012. NIH Image to ImageJ: 25 years of image analysis. Nature Methods 9: 671-675. 
Schultz ER, Zupanska AK, Sng NJ, Paul A-L, Ferl RJ. 2017. Skewing in Arabidopsis roots involves disparate environmental signaling pathways. BMC Plant Biology 17: 31.

Sedbrook JC, Carroll KL, Hung KF, Masson PH, Somerville C. 2002. The arabidopsis SKU5 gene encodes an extracellular glycosyl phosphatidylinositol-anchored glycoprotein involved in directional root growth. The Plant Cell 14: 1635-1648.

Shih H-W, Miller ND, Dai C, Spalding EP, Monshausen GB. 2014. The receptor-like kinase FERONIA is required for mechanical signal transduction in Arabidopsis seedlings. Current biology 24: 1887-1892.

\section{Soundappan I, Bennett T, Morffy N, Liang Y, Stanga JP, Abbas A, Leyser O, Nelson} DC. 2015. SMAX1-like/D53 family members enable distinct MAX2-dependent responses to strigolactones and karrikins in Arabidopsis. The Plant Cell 27: 3143-3159.

\section{Stanga JP, Smith SM, Briggs WR, Nelson DC. 2013. SUPPRESSOR OF MORE AXILLARY GROWTH2 1 Controls Seed Germination and Seedling Development in Arabidopsis. Plant Physiology 163: 318-330.}

Thompson MV, Holbrook NM. 2004. Root-gel interactions and the root waving behavior of Arabidopsis. Plant Physiology 135: 1822-1837.

Vaughn LM, Masson PH. 2011. A QTL study for regions contributing to Arabidopsis thaliana root skewing on tilted surfaces. Genes Genomes Genetics 1: 105-115.

Walton A, Stes E, Goeminne G, Braem L, Vuylsteke M, Matthys C, De Cuyper C, Staes A, Vandenbussche J, Boyer F-D, et al. 2016. The response of the root proteome to the synthetic strigolactone GR24 in Arabidopsis. Molecular \& Cellular Proteomics 15: 2744 2755.

Wang Y, Wang B, Gilroy S, Wassim Chehab E, Braam J. 2011. CML24 is involved in root mechanoresponses and cortical microtubule orientation in Arabidopsis. Journal of Plant Growth Regulation 30: 467-479.

Waters MT, Gutjahr C, Bennett T, Nelson DC. 2017. Strigolactone signaling and evolution. Annual Review of Plant Biology 68: 291-322.

Waters MT, Nelson DC, Scaffidi A, Flematti GR, Sun YK, Dixon KW, Smith SM. 2012. Specialisation within the DWARF14 protein family confers distinct responses to karrikins and strigolactones in Arabidopsis. Development 139: 1285-1295.

Waters MT, Scaffidi A, Flematti G, Smith SM. 2015a. Substrate-induced degradation of the a/b-fold hydrolase KARRIKIN INSENSITIVE2 requires a functional catalytic triad but is independent of MAX2. Molecular Plant 8: 814-817.

\section{Waters MT, Scaffidi A, Moulin SLY, Sun YK, Flematti GR, Smith SM. 2015b. A} Selaginella moellendorffii ortholog of KARRIKIN INSENSITIVE2 functions in Arabidopsis development but cannot mediate responses to karrikins or strigolactones. The Plant Cell 27: 1925-1944. 
Zhao L-H, Zhou XE, Yi W, Wu Z, Liu Y, Kang Y, Hou L, de Waal PW, Li S, Jiang Y, et al. 2015. Destabilization of strigolactone receptor DWARF14 by binding of ligand and E3ligase signaling effector DWARF3. Cell Research 25: 1219-1236.

667

668

669

670

671

672

673

674

675

676

677

678

679

680

681

682

683

684

685

686

687

688

689

690

691

692

693

694

695

\section{Zhou F, Lin Q, Zhu L, Ren Y, Zhou K, Shabek N, Wu F, Mao H, Dong W, Gan L, et al.} 2013. D14-SCFD3-dependent degradation of D53 regulates strigolactone signalling. Nature 504: 406-410.

Zwanenburg B, Mwakaboko AS, Reizelman A, Anilkumar G, Sethumadhavan D. 2009.

Structure and function of natural and synthetic signalling molecules in parasitic weed germination. Pest Management Science 65: 478-491.

\section{Figure legends:}

Fig.1. kai2 and max 2 mutants display an exaggerated rightward root skewing phenotype. A. Seedlings of kai2-1, kai2-2, $\max 2-7$, and $\max 2-8$, displayed an exaggerated rightward skew when grown at $90^{\circ}$. The scale bar represents $1 \mathrm{~cm}$. B. The root skewing angle $(\alpha)$ was measured as the deviation from the vertical for plants grown at a $90^{\circ}$ angle. C. The increased root skewing of karrikin-insensitive mutants measured as the simple deviation from the vertical could also be noted when measured as an increase in horizontal growth index (HGI) or (D) a decrease in vertical growth index (VGI). Data for each genotype are displayed as a beanplot with the skewing angle of individual roots shown as dark green horizontal lines, while the mean is represented by a thick black horizontal line. The estimated density of the distribution is illustrated by the shaded colour. The dashed line corresponds to the mean for the wild type. Positive values are rightward skews. * indicates significant difference compared to wild type (Tukey HSD, $p<0.05$ ). For each genotype, $n>65$ in 3 separate experiments.

Fig. 2. KAI2 and MAX2 regulate root skewing through the same pathway, which does not involve D14

A. Seedlings for the double mutant kai2-2/max2-8 showed no further increase in root skewing angle compared to kai2-2 (B). The scale bar indicates $1 \mathrm{~cm}$. Data for each genotype are displayed as a beanplot with the skewing angle of individual roots shown as dark green horizontal lines, while the mean is represented by a thick black horizontal line. The estimated density of the distribution is illustrated by the shaded colour. The dashed line corresponds to 
696

697

698

699

700

701

702

703

704

705

706

707

708

709

710

711

712

713

714

715

716

717

718

719

720

721

722

723

724

725

726

727

728

the mean for the wild type. * indicates significant difference compared to wild type (Tukey HSD, $p<0.05$ ). For each genotype, $n>66$ in 5 separate experiments. C. Seedlings for the SLinsensitive mutant Atd14 showed no increased rightward root skewing, and the measured skewing angle was not significantly different from that of the wild type (D). For each genotype, $n>73$ from 3 experiments.

\section{Fig. 3. Involvement of SMAX1 and SMXLs in root skewing.}

A. Seedlings for Col, $\max 2-1, \operatorname{smax} 2-1, \operatorname{smax} 2-1 / \max 2-1, \operatorname{smx} 16,7,8$ and $\operatorname{smx} 16,7,8 / \max 2-1$ showing root skewing while grown at $90^{\circ}$. The scale bar represents $1 \mathrm{~cm}$. B. Data for each genotype are displayed as a beanplot with the skewing angle of individual roots shown as dark green horizontal lines, while the mean is represented by a thick black horizontal line. The estimated density of the distribution is illustrated by the shaded colour. The dashed line corresponds to the mean for the wild type. * indicates a significant difference compared to wild type (Tukey HSD, $p<0.05$ ). For each genotype, $n>58$ from 6 experiments.

\section{Fig. 4. kai2 and $\max 2$ increased rightward root skewing when placed at $45^{\circ}$.}

A. Seedlings of kai2-1, kai2-2, $\max 2-7$, and $\max 2-8$, displayed an exaggerated rightward skew when grown vertically for six days then placed at $45^{\circ}$ for 3 days. The scale bar represents $1 \mathrm{~cm}$. B. The root skewing angle $(\alpha)$ was measured as the deviation from the vertical for plants grown at a $45^{\circ}$ angle for 3 days. C. Both $\max 2-8$ and kai2-2 mutants show increased cell file rotation (CFR) indicating that the root epidermal cells were twisting more compared to those of the wild type. CFR was measured as the number of epidermal cells that crossed a $1 \mathrm{~mm}$ line 1.5 to $2 \mathrm{~mm}$ from the root tip. Plants were grown at $45^{\circ}$. Data shown as mean $\pm \mathrm{SE}, n=28-42$ plants obtained in 4 separate experiments. Letters indicate significant differences (Tukey HSD, $p<$ 0.05 , except for the comparison Ler-max2-7 where the difference was significant at the $10 \%$ limit, pairwise $t$-test, $p<0.05$ ). The scale bar indicates $500 \mu \mathrm{m}$. D. The straightness (measured as the ratio of the chord Lc to root length L; Grabov et al., 2004; Vaughn \& Masson, 2011) of seedling roots from wild type, kai2-1 and kai2-2 decreased when plants were grown at $45^{\circ}$ compared to $90^{\circ}$ (shown in brackets behind genotype). Data for each genotype are displayed as a beanplot with the straightness of individual roots shown as dark green horizontal lines, while the mean is represented by a thick black horizontal line. The estimated density of the distribution is illustrated by the shaded colour. The dashed line corresponds to the mean for the wild type. ${ }^{*}$ indicates significant difference at the $5 \%$ level compared to wild type grown at 
$90^{\circ}$, while $\$$ indicates a significant difference to wild type grown at $45^{\circ}$. For each genotype, $n$ $>58$ in 3 separate experiments.

Fig. 5. kai2, max2, d14 mutants support normal transcriptional response to mechanostimulus.

Karrikin- and SL-insensitive mutants showed a normal response to mechanical stimulation at the transcript level. Nine day-old seedlings of wild type and mutants kai2-1 and kai2-2 (A), and $d 14$, $\max 2-1$ (B) were mechanically stimulated (MS) for 30 seconds, then collected 30 min later for transcript analysis of touch-sensitive genes CML12 and CML24, relative to housekeeping genes Tubulin 4 and Ubiquitin 10. The means of 6-9 replicates from 3 independent experiments are shown, each replicate based on the RNA extracted from roots of 30 to 40 seedlings. Data are shown as mean $\pm \mathrm{SE}$, letters indicate significant differences (Tukey HSD, $p<0.05)$.

Fig. 6. Mechano-stimulated $\left[\mathrm{Ca}^{2+}\right]_{\mathrm{cyt}}$ increase in $\max 2$ root tips. Individual excised root tips of $\mathrm{Col}$ and $\max 2$ expressing (apo)aequorin as a $\left[\mathrm{Ca}^{2+}\right]_{\mathrm{cyt}}$ reporter were mechanically stimulated by addition of buffer at $35 \mathrm{~s}$. The mean \pm SE of 40 to 67 roots in 5 independent trials are shown. Inset: Mean $\pm \mathrm{SE}$ maximal $\left[\mathrm{Ca}^{2+}\right]_{\mathrm{cyt}}$ increment in response to stimulus (peak response minus baseline).

\section{Fig. 7. Gravitropic response of kai2 is slower than wild type's.}

The tip orientation of roots from wild type and kai2-2 was recorded every $10 \mathrm{~min}$ and for $10 \mathrm{~h}$ after a change in gravitropic orientation. The change in tip orientation was normalised to the tip displacement to take into account differences in growth rate between genotype. Data are shown as mean $\pm \mathrm{SE}, n=16-22$ plants obtained in 5 experiments.

\section{Fig. 8. MAX2 regulation of root skewing involves SKS3 and SKU5}

A. Seedlings of Col, $\max 2-1$, sks3, sks3/max2-1, sku5, sku5/max2-1 mutants grown at $90^{\circ}$. The scale bar represents $1 \mathrm{~cm}$. B. Data for each genotype are displayed as a beanplot with the skewing angle of individual roots shown as dark green horizontal lines, while the mean is represented by a thick black horizontal line. The estimated density of the distribution is illustrated by the shaded colour. The dashed line corresponds to the mean for the wild type. * indicates a significant difference compared to wild type (Tukey HSD, $p<0.05$ ). For each genotype, $n>34$ in 3 separate experiments. 
764 Fig. S1. The root skewing angle of complemented lines of the kai2-2 mutant was reduced

765

766

767

768

769

770

771

772

773

774

775

776

777

778

779

780

781

782

783

784

785

786

787

788

789

790

791

792

793

794

795

796 compared to the kai2-2 mutant but remained higher than that of the Ler wild type (A). 10G and $12 \mathrm{H}$ are kai2-2 lines complemented by KAI2 expression under the native promoter. Data for each genotype are displayed as a beanplot with the skewing angle of individual roots shown as dark green horizontal lines, while the mean is represented by a thick black horizontal line. The estimated density of the distribution is illustrated by the shaded colour. The dashed line corresponds to the mean for the wild type. ${ }^{*}$ indicates a significant difference compared to wild type (Tukey HSD, $p<0.05$ ) while $\$$ indicates a significant difference compared to kai2-2 (Tukey HSD, $p<0.05$ ). For each genotype, $n>48$ in 4 separate experiments. (B) The root skewing angle of seedlings for three mutant alleles of $d l k 2$ showed no further increased compared to wild type. There is no significant difference between root skewing angle of $d l k 2$ alleles and wild type (Tukey HSD, n.s.). For each genotype, $n>98$ in 4 separate experiments.

Fig. S2. Effect of $\mathrm{KAR}_{2}$ on root skewing and primary root elongation in kai2 and $\operatorname{max2}$. Root skewing angle of Ler (A) and kai2-2 (C) plants grown under control conditions or in the presence of $2.5,5$ or $10 \mu \mathrm{M} \mathrm{KAR}_{2}$ in the medium and, $\max 2-8$ (E) grown under control conditions or in the presence of $5 \mu \mathrm{M} \mathrm{KAR}_{2}$ in the medium. Root elongation over a three-day period when Ler (B), kai2-2 (D) and $\max 2-8$ (F) plants were exposed to $\mathrm{KAR}_{2}$. Data for each genotype are displayed as a beanplot with the skewing angle of individual roots shown as dark green (or purple for the root elongation data) horizontal lines, while the mean is represented by a thick black horizontal line. The estimated density of the distribution is illustrated by the shaded colour. The dashed line corresponds to the mean for the control conditions. $*$ indicates a significant difference compared to control conditions (Tukey HSD, $p<0.05$ ). For each treatment and genotype combination, $n>64$ (except for kai2-2 under 2.5 and $10 \mu \mathrm{M}$ where $n$ $>30$ ) in at least 3 independent experiments.

Fig. S3. Effect of GR24 on root skewing in kai2, $\max 2$ and $d 14$. Root skewing angle of Col (A), max2-1 (B) and $d 14$ (C) plants grown under control conditions or in the presence of 1 or $5 \mu \mathrm{M}$ GR24 in the medium. Data for each genotype are displayed as a beanplot with the skewing angle of individual roots shown as dark green horizontal lines, while the mean is represented by a thick black horizontal line. The estimated density of the distribution is illustrated by the shaded colour. The dashed line corresponds to the mean for the control conditions. * indicates a significant difference compared to control conditions (Tukey HSD, $p$ 
$797<0.05)$. For each treatment and genotype combination, $n>86$ in at least 3 independent 798 experiments.

799

800 Fig. S4. sks3 and sku5 do not suppress the high lateral root density in max 2

801 Lateral roots per cm of primary roots in 9-d-old seedlings. Data are shown as mean \pm SE. For

802 each genotype, $n>51$ plants grown in 5 separate experiment. Letters indicate significant 803 differences (Tukey HSD, $p<0.05$ ).

804

805 Fig. S5. sks3 does not suppress low germination rate in max2

806 Seeds were germinated on $0.8 \%(\mathrm{w} / \mathrm{v})$ agar plates and germination rate was scored after $72 \mathrm{~h}$.

807 Data are shown as mean \pm SE, for 10 batches of seeds each batch holding $>80$ seeds. $\bullet$ Indicates 808 significant difference compare to the wild type (Tukey HSD, $p<0.1$ ).

809

810 Fig. S6. Root diameter of max2-7 and kai2-2 plants was lower than that of wild type. Letters

811 indicate statistical significance at the 1\% level (Tukey, HSD). Data shown as mean \pm SE, $n>$ 81236 per genotype in a total of 5 experiments.

813 
Figure 1

A

B
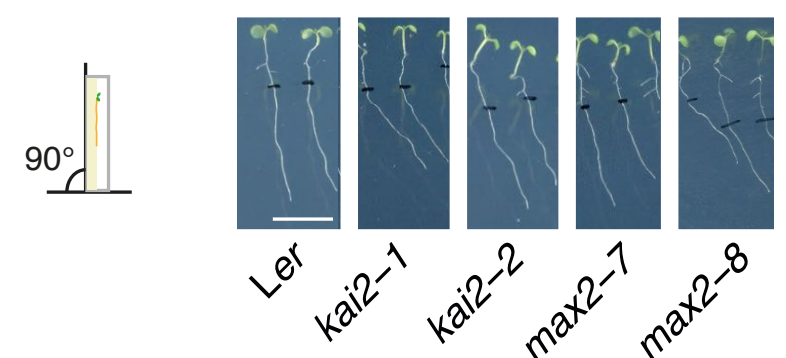

2

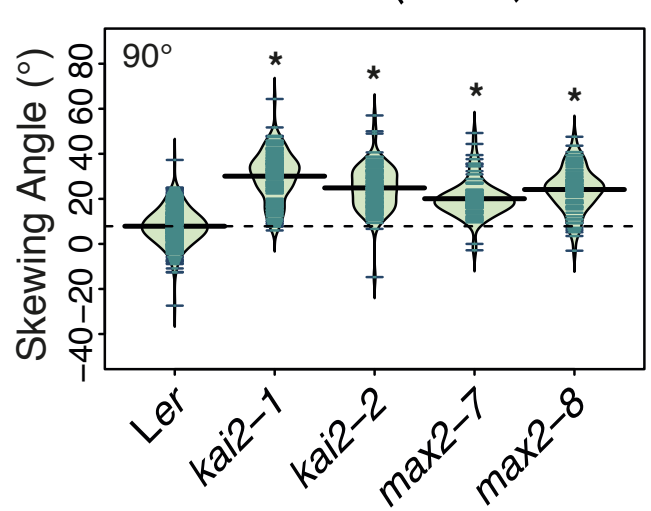

D

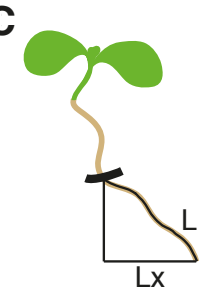

$H G I=L x / L$

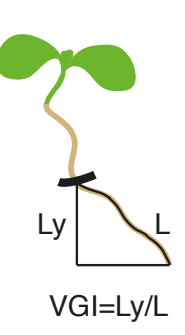

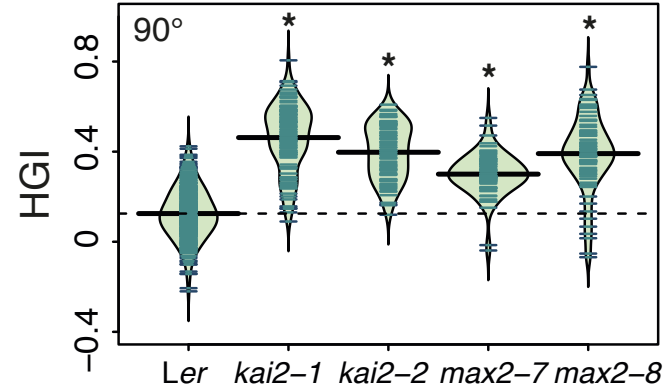

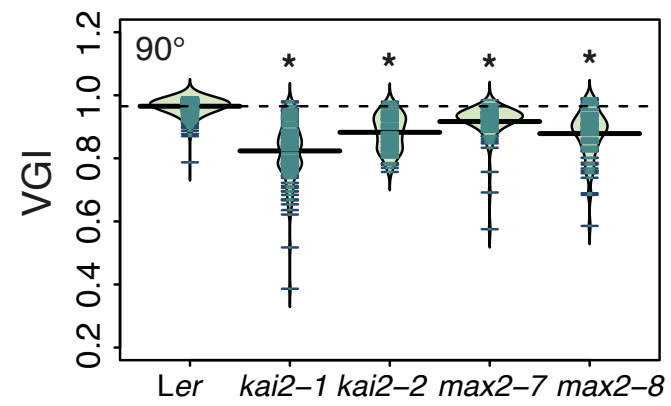


Figure 2

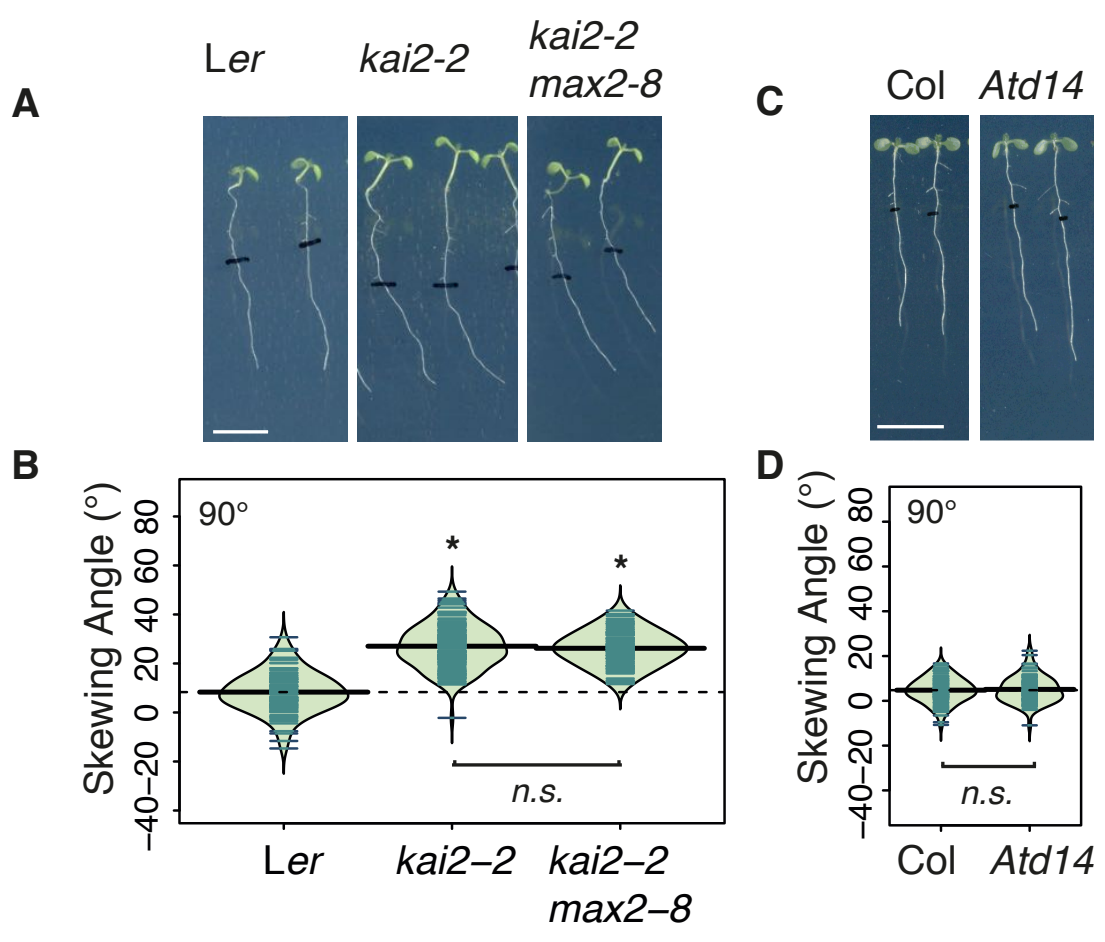


Figure 3

A
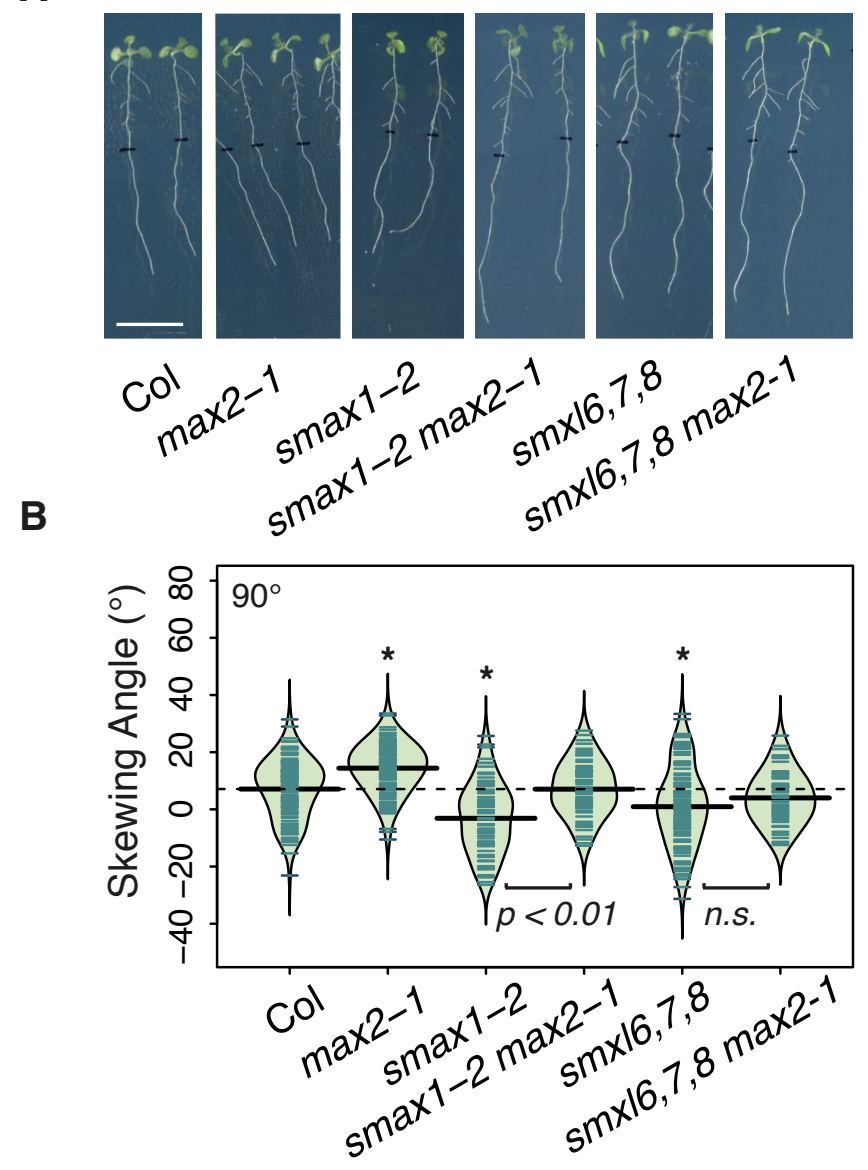
Figure 4

A

B
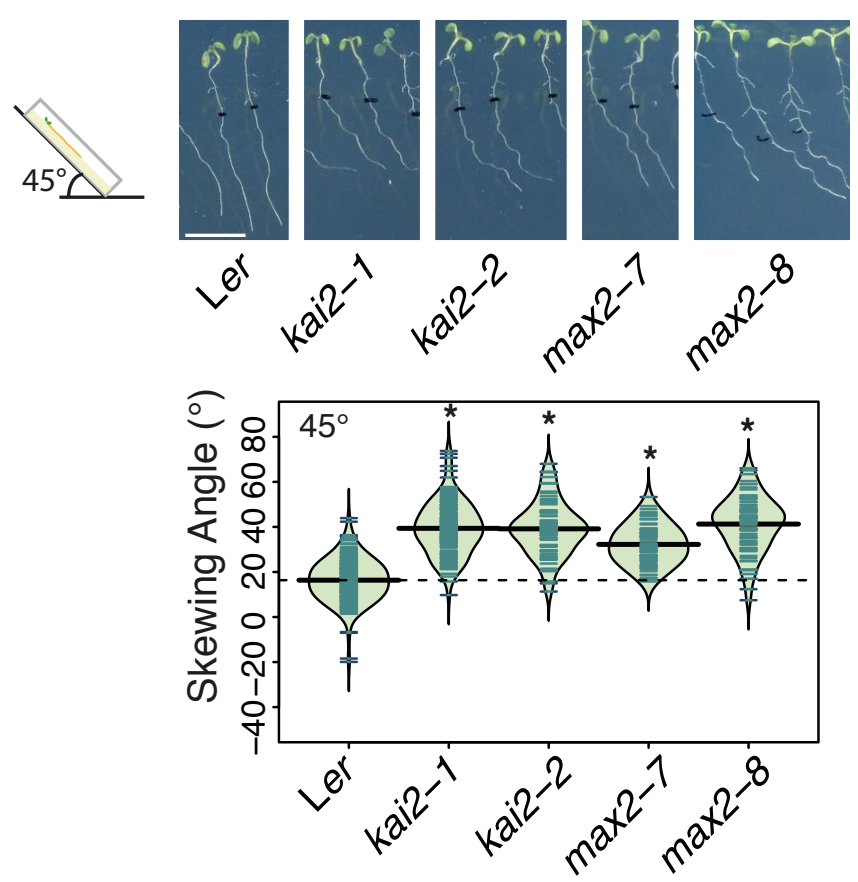

D

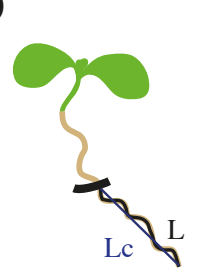

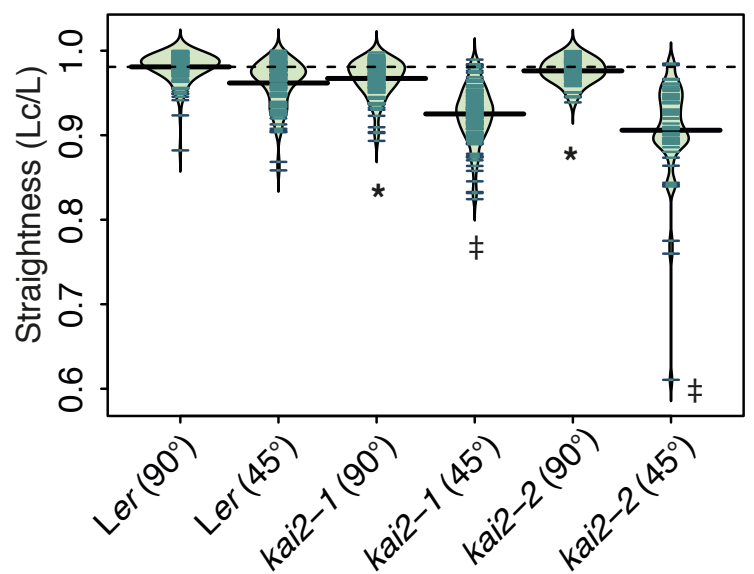
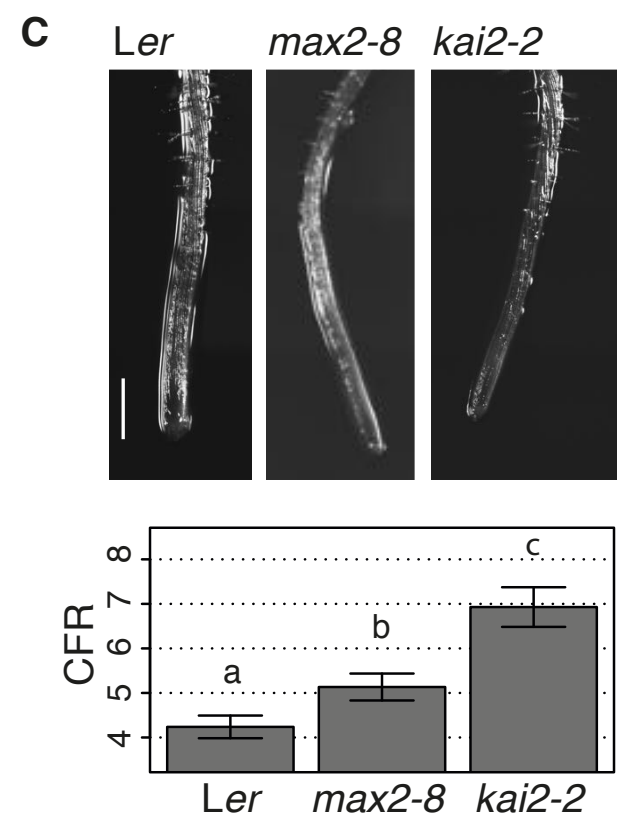
Figure 5

A
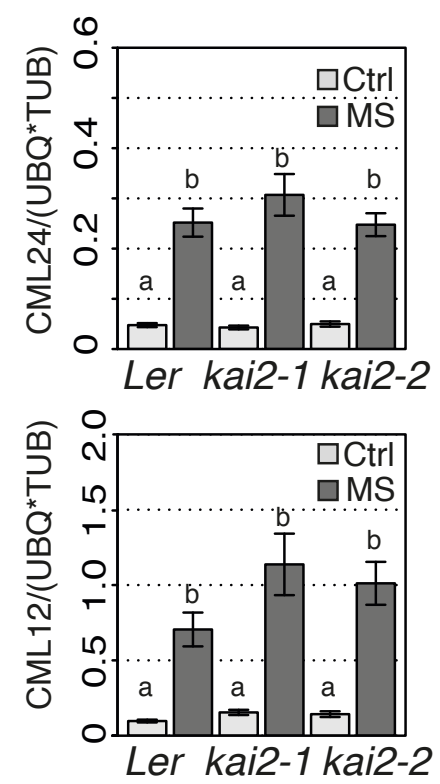

B
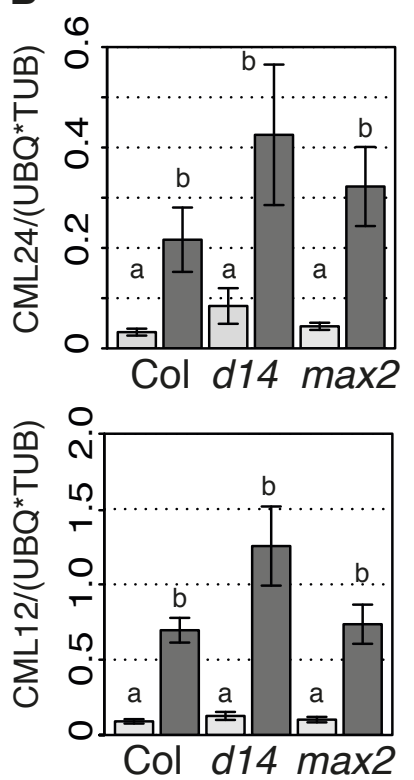
Figure 6

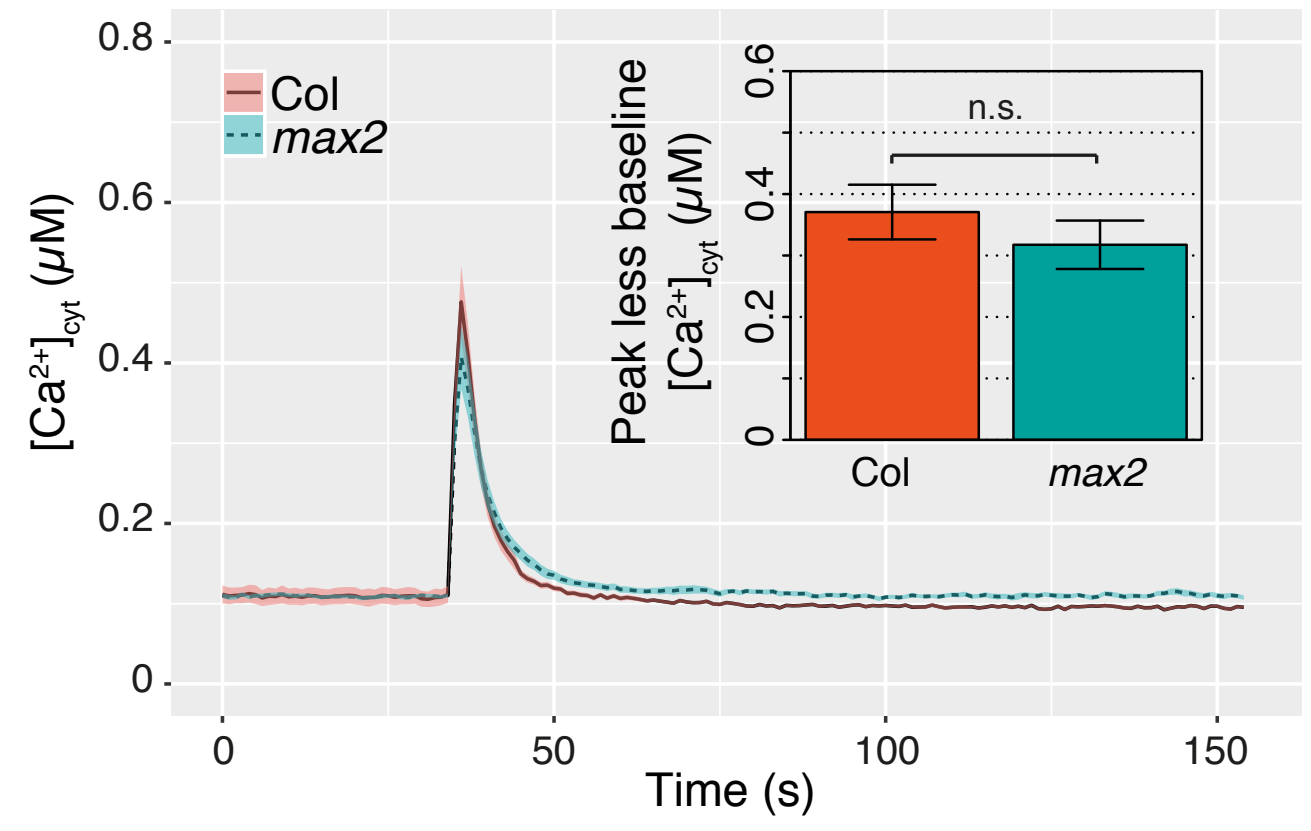


Figure 7

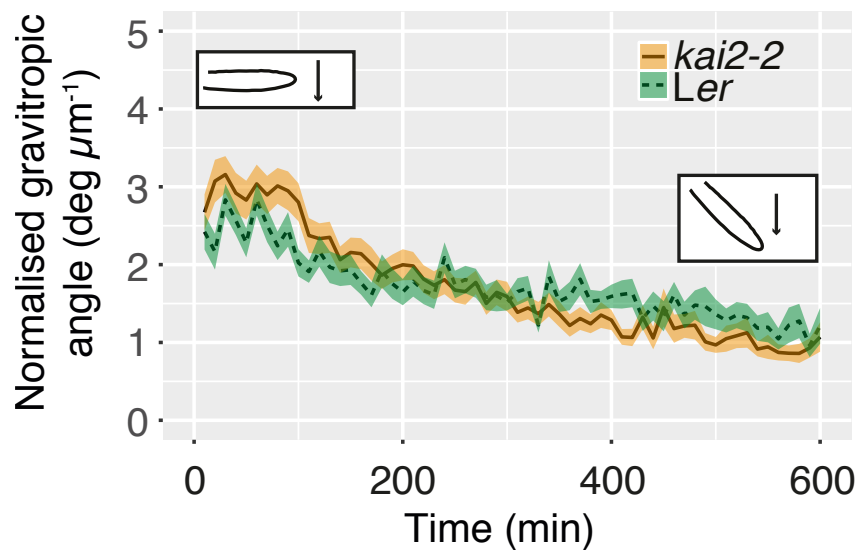


Figure 8

A
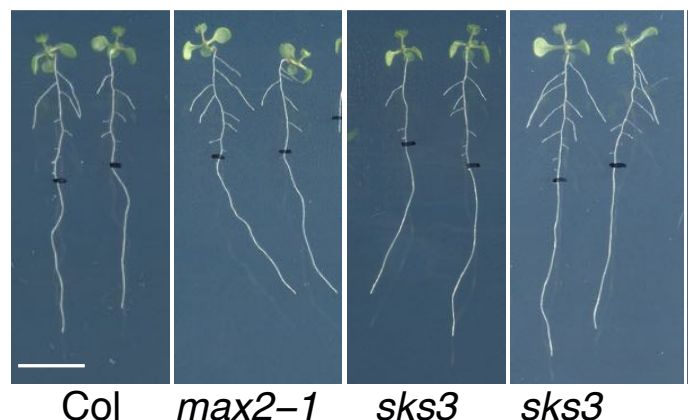

sks3
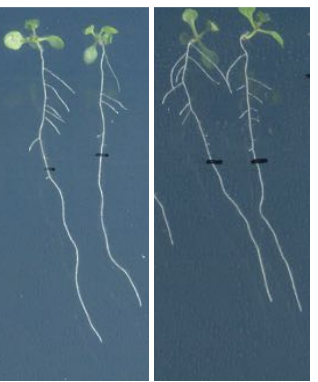

$\max 2-1$

sku5

sku5

$\max 2-1$

B

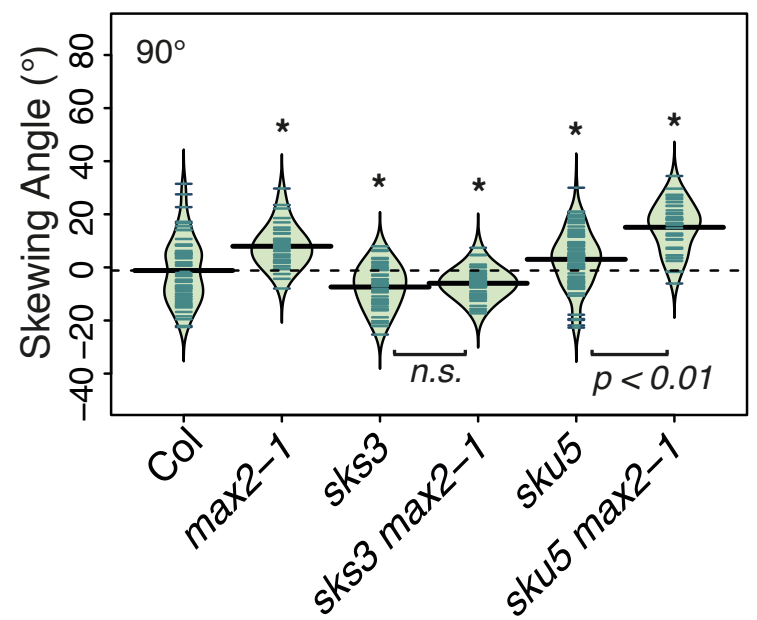

